Japan. J. Math.

Vol. 11, No. 1, 1985

\title{
Irreducible representations of a class of unbounded operator algebras
}

\author{
By Subhash J. BhatT
}

(Received May 1, 1984)

Atsushi Inoue [7] has initiated the study of certain algebras of unbounded operators called $E C^{*}$-algebras and $E W^{*}$-algebras. These algebras occur naturally in the analysis of unbounded generalization of left Hilbert algebras [10]. The purpose of this note is to examine irreducible representations of these algebras in the context of unbounded representation theory [12].

Definitions. (a) Let $\mathscr{D}$ be a dense subspace of a Hilbert space $\mathscr{K}$. A collection $\mathscr{U}$ of linear operators, not necessarily bounded, all defined on $\mathscr{D}$ is called an $E C^{*}$-algebra on $\mathscr{D}$ over $\mathscr{U}_{b}$ if

(i) $\mathscr{U}$ is an algebra under the operations of (usual) sum, product and scalar multiplications.

(ii) $T \rightarrow T^{*}=\left.T^{\#}\right|_{\mathscr{D}}$ defines an involution on $\mathscr{U}$ where $\left.T^{\#}\right|_{\mathscr{g}}$ is the restriction to $\mathscr{D}$ of the operator adjoint $T^{\#}$ of $T$ in $\mathscr{K}$.

(iii) $\overline{\mathscr{U}}_{b}=\{\bar{T} \mid T \in \mathscr{U}$ is bounded $\}$ is a $C^{*}$-algebra, bar denoting the closure of the operator.

(iv) For each $T \in \mathscr{U},\left(1+T^{*} T\right)^{-1} \in \mathscr{U}$.

Further if $\overline{\mathscr{U}}_{b}$ is a $W^{*}$-algebra, then $\mathscr{U}$ is called an $E W^{*}$-algebra.

(b) A representation $(\pi, \mathscr{D}(\pi), \mathscr{H})$ of $a^{*}$ algebra $A$ is a mapping $\pi$ of $A$ into linear operators (not necessarily bounded) all defined on a common domain $\mathscr{D}(\pi)$ dense in a Hilbert space $\mathscr{H}$ such that for all $x, y \in A ; \alpha, \beta \in C$, $\xi, \eta \in \mathscr{D}(\pi)$, the following hold.

1. $\pi(\alpha x+\beta y) \xi=\alpha \pi(x) \xi+\beta \pi(y) \xi$

2. $\pi(x) \mathscr{D}(\pi) \subset \mathscr{D}(\pi)$ and $\pi(x y) \xi=\pi(x) \pi(y) \xi$.

If $A$ has identity 1 , then

3. $\pi(1)=1$.

Further $\pi$ is called hermitian if

4. $\langle\pi(x) \xi, \eta\rangle=\left\langle\xi, \pi\left(x^{*}\right) \eta\right\rangle$. 
If $\pi(x)$ is a bounded operator for every $x$, then $\pi$ is called a bounded representation.

For basic theory, we refer to the references mentioned above. Throughout, all the representations are hermitian.

Theorem 1. Let $\mathscr{U}$ be an EC*-algebra defined on a linear subspace $\mathscr{D}$ dense in a Hilbert space $\mathscr{K} . \quad$ Let $(\pi, \mathscr{D}(\pi), \mathscr{H})$ be a closed representation of $\mathscr{U}$ on $\mathscr{H}$. If the only self-adjoint invariant subspaces for $\pi$ are $\{0\}$ and $\mathscr{D}(\pi)$, then $\mathscr{D}(\pi)=\mathscr{H}$ and $\pi$ is a bounded representation.

The proof required the techniques of $G B^{*}$-algebra [6]. By [6, Theorem 7.12] an $E C^{*}$-algebra $\mathscr{U}$ is a concrete realization of a locally convex $G B^{*}$ algebra. Various $G B^{*}$-topologies carried by $\mathscr{U}$ are discribed in [7, (I), §2]. Further by $[6, \S 6] \mathscr{U}$ carries a largest locally convex $G B^{*}$-topology (called the Dixon topology and denoted throughout by $\tau$ ) having the $C^{*}$-algebra $\mathscr{U}_{b}$ $=\{T \in \mathscr{U} \mid T$ is bounded $\}$ as the bounded part. The topology $\tau$ is known to be barrelled [6, Lemma 6.2] with the result the multiplication in $(\mathscr{U}, \tau)$ is hypocontinuous [6, Lemma 6.3], and hence sequentially jointly continuous. We shall need the following [3].

Theorem A. Let $\mathscr{U}$ be an $E C^{*}$-algebra and $t$ be any locally convex $G B^{*}$ topology on $\mathscr{U}$. Then $\mathscr{U}_{b}$ is sequentially dense in $\mathscr{U}$.

In fact, as discussed in [4], for each $T \in \mathscr{U}, T_{n}=T\left(1+(1 / n) T^{*} T\right)^{-1} \in \mathscr{U}_{b}$ for each $n=1,2, \cdots$ and $T_{n} \rightarrow T$ in $\tau$. We shall also need the following version of [10, Theorem 4.1].

Theorem B. Let $\mathscr{U}$ be an EC*-algebra. Let $(\pi, \mathscr{D}(\pi), \mathscr{H})$ be a representation of $\mathcal{U}$. Let $\sigma_{\pi}$ be the weak topology on $\pi(\mathscr{U})$ defined by the semi-norms $\pi(T) \rightarrow\langle\pi(T) \xi, \eta\rangle$ for $\xi, \eta$ in $\mathscr{D}(\pi)$. Then $\pi:(\mathscr{U}, \tau) \rightarrow\left(\pi(\mathscr{U}), \sigma_{\pi}\right)$ is continuous.

Proof of the Theorem. Let $\rho(T)=\overline{\pi(T)}\left(T \in \mathscr{U}_{b}\right)$. Then for each such $T$, the domain of $\rho(T)$ is $\mathscr{D}(\rho(T))=\mathscr{H}$ and $\rho(T) \in \beta(\mathscr{H})$. We show that $\rho$ is (topologically) irreducible (in the usual sense of $C^{*}$-representation theory).

Suppose that $\mathscr{H}_{1}$ is a norm closed subspace of $\mathscr{H}$ such that $\mathscr{H}_{1} \neq(0)$, $\mathscr{H}_{1} \neq \mathscr{H}$ and $\rho\left(\mathscr{U}_{b}\right) \mathscr{H}_{1} \subset \mathscr{H}_{1}$. Let $\mathscr{D}_{1}=\left\{\xi \in \mathscr{D}(\pi) \mid \rho(T) \xi \in \mathscr{H}_{1}\right.$ for all $\left.T \in \mathscr{U}_{b}\right\}$. Obviously, $\pi\left(\mathscr{U}_{b}\right) \mathscr{D}_{1} \subset \mathscr{D}_{1}$. We claim that $\pi(\mathscr{U}) \mathscr{D}_{1} \subset \mathscr{D}_{1}$. Indeed, let $\xi \in \mathscr{D}_{1}, T \in \mathscr{U}$. Then $\pi(T) \xi \in \mathscr{D}_{1}$ if for all $S \in \mathscr{U}_{b}, \rho(S) \pi(T) \xi=\pi(S T) \xi \in \mathscr{H}_{1}$. By Theorem A, $\mathrm{T}_{n} \rightarrow T$ for some sequence $\left(T_{n}\right)$ in $\mathscr{U}_{b}$. By the sequential joint continuity of multiplication, $\left(T-T_{n}\right) * S * S\left(T-T_{n}\right) \rightarrow 0$. Therefore, by Theorem B,

$$
\left\|\pi(S T) \xi-\pi\left(S T_{n}\right) \xi\right\|^{2}=\left\langle\pi\left(T-T_{n}\right) * S * S\left(T-T_{n}\right) \xi, \xi\right\rangle \rightarrow 0 .
$$


But $\pi\left(S T_{n}\right) \xi \in \mathscr{H}_{1}$ and $\mathscr{H}_{1}$ is norm closed, hence $\pi(S T) \xi \in \mathscr{H}_{1}$ giving $\pi(\mathscr{U}) \mathscr{D}_{1} \subset \mathscr{D}_{1}$. Further, $\mathscr{D}_{1}$ is a closed subspace of $\left(\mathscr{D}(\pi), t_{\mathscr{U}}\right)$ where $t_{\mathscr{L}}$ is the induced topology $[11, \S 2]$ on $\mathscr{D}(\pi)$ defined by the semi-norms $\xi \rightarrow\|\pi(A) \xi\|(A \in \mathscr{U})$. For, let $\xi \in \mathscr{D}(\pi)$ be such that for some net $\left(\xi_{\alpha}\right)$ in $\mathscr{D}_{1}, \xi_{\alpha} \rightarrow \xi$ in $t_{\mathscr{U}}$. Then for each $T \in \mathscr{U}$, $\left\|\pi(T)\left(\xi_{\alpha}-\xi\right)\right\| \rightarrow 0$. But by above, for each $T \in \mathscr{U}_{b}, \pi(T) \xi_{\alpha} \in \mathscr{D}_{1} \subset \mathscr{H}_{1}$ and $\mathscr{H}_{1}$ is norm closed, hence $\pi(T) \xi \in \mathscr{H}_{1}$. Thus $\xi \in \mathscr{D}_{1}$. Now $\pi$ being a closed representation of the symmetric ${ }^{*}$ algebra $\mathscr{U}$, it is self-adjoint by [8, Lemma 3.5]. Hence, an in [11, Theorem 4.7], it follows that $\mathscr{D}_{1}$ is a self-adjoint $\pi$-invariant subspace of $\mathscr{D}(\pi)$. Hence by the hypothesis, $\mathscr{D}_{1}=(0)$ or $\mathscr{D}_{1}=\mathscr{D}(\pi)$. In the former case, $\mathscr{H}_{1}=(0)$; in the latter one, $\mathscr{H}_{1}=\mathscr{H}$. In any case, it follows that $\rho$ is topologically irreducible.

Now by the well known result of Kadison [12, Corollary 1.12.17], $\rho$ is algebraically irreducible. Hence, as $\rho\left(\mathscr{U}_{b}\right) \mathscr{D}(\pi) \subset \mathscr{D}(\pi)$, we get $\mathscr{D}(\pi)=\mathscr{H}$. Now the closed graph theorem implies that $\pi$ is bounded.

Thus if $(\pi, \mathscr{D}(\pi), \mathscr{H})$ is a closed (algebraically) irreducible representation of an $E C^{*}$-algebra $\mathscr{U}$, then $\mathscr{D}(\pi)=\mathscr{H}$ and $\pi$ is bounded; and further, if $\mathscr{U}$ is commutative, then $\pi$ is one dimensional. Theorem 1 does not hold for arbitrary algebra; e.g. take $\mathscr{H}=L^{2}(R), \mathscr{D}=\mathscr{S}(\boldsymbol{R})$, the Schwartz space of rapidly decreasing $C^{\infty}$-functions on $R$. Let $\mathscr{U}$ be the complex algebra with identity generated by the position-momentum operators $p(f)=i(d f / d t), q(f)(t)$ $t f(t)(t \in R, f \in \mathscr{D})$. Let $*$ be the unique involution on $\mathscr{U}$ making $p$ and $q$ selfadjoint. Then the identity representation of $\mathscr{U}$ is known [11] to be selfadjoint having no non-trivial self-adjoint invariant subspaces. It also follows that unlike $C^{*}$-algebras, an $E C^{*}$-algebra need not admit any irreducible representation at all e.g. consider the $E C^{*}$-algebra $\mathscr{U}$ obtained by taking the faithful Dixon representation [6, Theorem 7.1] of the *algebra $L^{w}[0,1]=$ $\cap_{1<p \leqslant \infty} L^{p}[0,1]$ with the pointwise operations [1, Example 3]. Next we discuss several conditions ensuring the existence of irreducible representations.

Corollary 2. Let $\mathscr{U}$ be an EC*-algebra. The following are equivalent.

(a) There exists a closed irreducible representation of $\mathscr{U}$.

(b) There exists a $C^{*}$-semi-norm on $\mathscr{U}$.

(c) There exists a non-zero bounded representation of $\mathscr{U}$.

Proof. That $(\mathrm{a}) \Rightarrow(\mathrm{c}) \Rightarrow(\mathrm{b})$ are immediate in view of Theorem 1 . Let $p$ be a $C^{*}$-semi-norm on $\mathscr{U}$. Let $\mathbf{I}=\operatorname{ker} p, a^{*}$ ideal in $\mathscr{U}$. Let $\tilde{\mathscr{U}}=\mathscr{U} / \mathbf{I}$ and $\tilde{p}$ be the $C^{*}$-norm on induced by $p$. We show that $(\tilde{\mathscr{U}}, \tilde{p})$ is complete (this seems to be of some independent interest). First note that that for each $\left.T \in \mathscr{U}, p\left(1+(1 / n) T^{*} T\right)^{-1}\right) \leq 1$. (Pass to the quotient, complete it and use the $C^{*}$-result.) Now let $\mathbf{J}=\left\{T \in \mathscr{U}_{b} \mid p(T)=0\right\}=\mathscr{U}_{b} \cap \mathbf{I}$. As $\mathscr{U}_{b}$ is a $C^{*}$-algebra, $p$ is

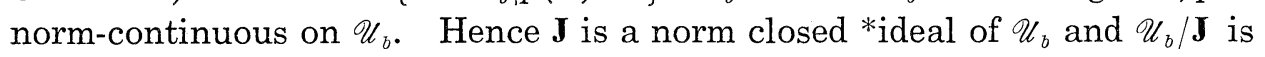


a $C^{*}$-algebra under the quotient norm $\|\cdot\|$. But $T+\mathbf{J} \rightarrow \tilde{p}(T+\mathbf{J})$ is also a $C^{*}$-norm (not necessarily complete a priori) on $\mathscr{U}_{b} / \mathbf{J}$. Again by the standard $C^{*}$-arguments. $\quad p(T)=\tilde{p}(T+\mathbf{J})=\|T+\mathbf{J}\|\left(T \in \mathscr{U}_{b}\right) . \quad$ Thus $\phi: T+\mathbf{J} \rightarrow T+\mathbf{I}$ is an isometric embedding of $\mathscr{U}_{b} / \mathbf{J}$ into $(\tilde{\mathscr{U}}, \tilde{p})$ with the result $\phi\left(\mathscr{U}_{b} / \mathbf{J}\right)$ is closed in $(\tilde{\mathscr{U}}, \tilde{p})$. On the other hand, for each $T \in \mathscr{U}, T\left(1+(1 / n) T^{*} T\right)^{-1} \in \mathscr{U}_{b}$ and $\lim _{n} T\left(1+(1 / n) T^{*} T\right)^{-1}=T$ in $\tau$. Also,

$$
\begin{aligned}
p\left(T-T\left(1+\frac{1}{n} T^{*} T\right)^{-1}\right) & =p\left(T\left(\frac{T^{*} T}{n}\right)\left(1+\frac{1}{n} T^{*} T\right)^{-1}\right) \\
& \leq \frac{1}{n} p(T) p\left(T^{*} T\right) \rightarrow 0 \text { as } n \rightarrow \infty .
\end{aligned}
$$

Thus $T\left(1+(1 / n) T^{*} T\right)^{-1}+\mathbf{I} \rightarrow T+\mathbf{I}$ in $(\tilde{\mathscr{U}}, \tilde{p})$. Therefore, $\phi\left(\mathscr{U}_{b} / \mathbf{J}\right)$ is dense in $(\tilde{\mathscr{U}}, \tilde{p})$. It follows that $(\tilde{\mathscr{U}}, \tilde{p})$ is a $C^{*}$-algebra. Now it is easily seen that every irreducible representation of $\tilde{\mathscr{U}}$ lifts to an irreducible representation of $\mathscr{U}$, and (a) follows from (b). This completes the proof.

The existence of a faithful family of irreducible representations greatly simplifies the structure of an $E C^{*}$-algebra, namely, makes it a pre $b^{*}$ algebra [6, Example 3.2] under a suitable topology. A weakly unbounded $E C^{*}$-algebra [9] $\mathscr{U}$ is a *subalgebra of the product II $\mathscr{U}_{\alpha}$ [7 (I), Theorem 3.7] of a family $\left(\mathscr{U}_{\alpha}\right)$ of $C^{*}$-algebras such that $\overline{\mathscr{U}}_{b}=\left\{\bar{T} \mid T \in \mathscr{U}_{b}\right\}=\Sigma^{\oplus \mathscr{U}_{\alpha}}$, the usual $C^{*}$-direct sum. It follows from Corollary (2) that an $E C^{*}$-algebra $\mathscr{U}$ admits a faithfully family of irreducible representations, if and only if $\mathscr{U}$ is (*isomorphic to) a weakly unbounded $E C^{*}$-algebra.

Proposition 3. Let $\mathscr{U}$ be an EW*-algebra. Let $\sigma$ be the weak topology on $\mathscr{U}$ defined by the semi-norms $T \rightarrow|\langle T \xi, \eta\rangle|$ for $\xi, \eta \in \mathscr{D}$. The following are equivalent.

(a) $\mathscr{U}$ admits a weakly continuous irreducible representation.

(b) $\mathscr{U}_{b}$ admits a non-zero minimal projection.

The proof requires the following lemma which is of some independent interest. The proof is a modification of standard $W^{*}$-techniques.

Lemma 4. Let $\mathscr{U}$ be an $E W^{*}$-algebra. A subset $\mathbf{I}$ of $\mathscr{U}$ is a $\sigma$-closed left ideal of $\mathscr{U}$ if and only if there is a unique projection $P$ in $\mathscr{U}$ such that $\mathbf{I}=\mathscr{U} P$. Further

(a) I is two sided if and only if $P$ is a central projection.

(b) I is maximal if and only if $1-P$ is a minimal projection.

Proof of the Lemma. As in [7 (I), § 2], $(\mathscr{U}, \sigma)$ is a locally convex $G B^{*-}$ algebra with bounded part $\mathscr{U}_{b}$. By Theorem $\mathrm{A}, \mathscr{U}_{b}$ is $\sigma$-dense in $\mathscr{U}$. Now 
let $\mathbf{I}$ be a $\sigma$-closed left ideal of $\mathscr{U}$. Then $\mathbf{L}=\mathbf{I} \cap \mathscr{U}_{b}$ is a $\sigma_{w}$-closed left of the $W^{*}$-algebra $\mathscr{U}_{b}$ (Here $\sigma_{w}$ is the weak topology on $\mathscr{U}_{b}$ as the dual of a Banach space). Then by the $\sigma$-continuity of the involution on $\mathscr{U}, \mathbf{L} \cap \mathbf{L}^{*}=\mathbf{I} \cap \mathbf{I}^{*} \cap \mathscr{U}_{b}$ is a $\sigma_{w}$-closed two sided ideal of $\mathscr{U}_{b}$. Thus it is a $W^{*}$-algebra in its own right admitting an identity $P$ that is a projection in $\mathscr{U}_{b}$. Next, as in [12, Proposition 1.10], we have $\mathbf{I} \cap \mathscr{U}_{b}=\mathscr{U}_{b} P=\left\{T \in \mathscr{U}_{b} \mid T=T P\right\}$. This gives $\mathbf{I}=\mathscr{U} P$. In fact, given $T \in I, T_{n}=T\left(1+(1 / n) T^{*} T\right)^{-1} \in \mathbf{I} \cap \mathscr{U}_{b}$ and $T_{n} P=T_{n} \rightarrow T=T P$. The converse, as well as the remaining assertions, are immediate.

Proof of the Proposition. (a) $\Rightarrow(\mathrm{b})$. If $(\pi, \mathscr{D}(\pi), \mathscr{H})$ is a $\sigma-\sigma_{\pi}$ continuous irreducible representation of $\mathscr{U}$, then for each $\xi \neq 0$ in $\mathscr{D}(\pi), \mathbf{L}=$ $\{T \in \mathscr{U} \mid \pi(T) \xi=0\}$ is a maximal left ideal that is $\sigma$-closed. The lemma gives (b).

Conversely, let $P$ be a minimal projection in $\mathscr{U}_{b}$. Then $\mathbf{I}=\mathscr{U}(1-P)$ is a $\sigma$-closed maximal left ideal, and $P \mathscr{U}_{b} P \simeq C$. By theorem $\mathrm{A}, P \mathscr{U} P \simeq C$ and $f: T$ $\rightarrow$ PTP define a linear functional on $\mathscr{U}$ that is easily seen to be positive using [7 (1), Proposition 2.11]. Let $\mathbf{L}^{\prime}=\left\{T \mid f\left(T^{*} T\right)=0\right\}$. Then, if $T=S(1-P) \in L$, $f\left(T^{*} T\right)=P(1-P) S^{*} S(1-P) P=0$ and so $\mathbf{L} \subset \mathbf{L}^{\prime}$. Since $\mathbf{L}$ is maximal, $\mathbf{L}=\mathbf{L}^{\prime}$. Let $(\pi, \mathscr{D}(\pi), \mathscr{H})$ be the closed strongly cyclic representation of $\mathscr{U}$ constructed from $f$ by the GNS construction. Then, as $f$ is $\sigma$-continuous, $\pi$ is $\sigma-\sigma_{\pi}$ continuous; and by the standard arguments, it is irreducible. Thus $(b) \Rightarrow(a)$ and the proposition follows.

Thus an $E W^{*}$-algebra $\mathscr{U}$ admits a faithfully family of weakly continuous irreducible representations, if and only if $\mathscr{U}_{b}$ is an atomic $W^{*}$-algebra.

Acknowledgement. Thanks are due to Dr. Atshushi Inoue of Fukuoka, Japan for sending the preprints of his work long before their publications.

\section{REFERENCES}

[1] G. R. Allan, On a class of locally convex algebras, Proc. London Math. Soc., 17 (1967), 91-114.

[2] W. Arveson, An Invitation to $C^{*}$-algebras, Springer-Verlag, Berlin, 1973.

[ 3 ] S. J. Bhatt, A note on Generalized $B^{*}$-algebras, J. Indian Math. Soc., 43 (1979), $253-257$.

[4] S. J. Bhatt, On Jordan representations of unbounded operator algebras, Proc. Amer. Math. Soc., 84 (1982), 393-394.

[5] S. J. Bhatt, Representability of positive functionals on abstract *algebras without identity with applications to locally convex algebras, Yokohama J. Math., 29 (1981), 7-16.

[ 6 ] P. G. Dixon, Generalized $B^{*}$-algebras, Proc. London Math. Soc., 21 (1970), 693715 . 
[ 7 ] A. Inoue, On a class of unbounded operator algebras, I, II, III; Pacific J. Math., 65 (1976), 36-57; ibid 66 (1976), 411-431; ibid 69 (1977), 105-115.

[8] A. Inoue, Unbounded representations of symmetric *algebras, J. Math. Soc. Japan, 29 (1977) , 219-232.

[9] A. Inoue, Weakly unbounded operator algebras, Mem. Fac. Sci. Kyushu Univ. Ser. A, 31 (1977), 267-284.

[10] A. Inoue, Unbounded generalizations of left Hilbert algebras, I, II, Jr. Functional Analysis, 34 (1979), 339-362, ibid, 35 (1980), 230-250.

[11] G. Laßner, Topological algebras of operators, Rep. Mathematical Phys., 3 (1972), 279-293.

[12] R. T. Powers, Self-adjoint algebras of unbounded operators, Comm. Math. Phys., 21 (1971), 85-124.

[13] S. Sakai, $C^{*}$-and $W^{*}$-algebras, Springer-Verlag, Berlin, 1971.

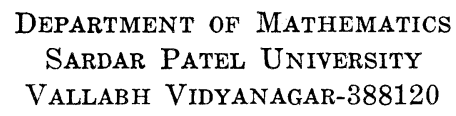

